\title{
A National Survey on Patient Provider Agreements When Prescribing Opioids for Chronic Pain
}

\author{
Jordana Laks, MD, MPH®, Daniel P. Alford, MD, MPH, Krupa Patel, MD, Margaret Jones, \\ Emily Armstrong, Katherine Waite, Lori Henault, MPH, and Michael K. Paasche-Orlow, \\ $M D, M A, M P H$
}

Section of General Internal Medicine, Department of Medicine, Boston University School of Medicine and Boston Medical Center, Boston, MA, USA.

BACKGROUND: Many national guidelines recommend the use of patient provider agreements (PPAs) when prescribing opioids for chronic pain. There are no standards for PPA content, readability, or administration processes. OBJECTIVE: Conduct a national survey of providers who use PPAs to describe the process of administering them, assess views on their utility, and obtain PPAs to evaluate thematic content and readability.

DESIGN: Cross-sectional electronic survey and request for PPAs.

PARTICIPANTS: Registrants for the Safer/Competent Opioid Prescribing Education (SCOPE of Pain) Program between March 2013 and June 2017.

MAIN MEASURES: Respondents' reports on how PPAs are administered and views on their usefulness. A sample of PPAs assessed for themes and readability.

KEY RESULTS: Using a convenience sample of 62,530 SCOPE of Pain registrants, we obtained a cohort of 430 individuals from 43 states who use PPAs. The majority of respondents worked in primary care (64\%) and pain (18\%) specialties. Reviewing PPAs with patients was primarily done by prescribers (80\%), and the average perceived time to administer PPAs was 13 min. Although 66\% of respondents thought PPAs were "often" or "always" worth the effort, only $28 \%$ considered them "often" or "always" effective in reducing opioid misuse. The PPA reading burden surpassed recommended patient education standards, with only $2.5 \%$ at or below fifth-grade reading level. PPAs focused more on rules and consequences of patients' noncompliance than on a shared treatment plan.

CONCLUSIONS: Most respondents perceive patient provider agreements (PPAs) as time-consuming and minimally effective in reducing opioid misuse yet still view them as valuable. PPAs are written far above recommended reading levels and serve primarily to convey consequences of non-compliance. Because PPAs are recommended by national safer opioid prescribing guidelines as a risk mitigation strategy, it would be beneficial to develop a standard PPA and study its effectiveness.

Preliminary study results were presented at the Society of General Internal Medicine annual meeting in Denver, CO, in April 2018; the 6th International Conference on Communication in Healthcare in Porto, Portugal, in September 2018; and the 42nd annual meeting of the Association for Multidisciplinary Education and Research in Substance use and Addiction in San Erancisco. CA. in November 2018

Received February 21, 2020

Accepted November 25, 2020

Published online January 8, 2021
KEY WORDS: patient provider agreements; chronic pain; opioids.

J Gen Intern Med 36(3):600-5

DOI: $10.1007 / \mathrm{s} 11606-020-06364-2$

(C) Society of General Internal Medicine 2021

\section{INTRODUCTION}

In the United States, increased opioid prescribing has been associated with a rise in opioid-related morbidity and mortality. ${ }^{1}$ In response, national organizations including the Centers for Disease Control and Prevention (CDC) and Department of Veterans Affairs have disseminated safer opioid prescribing guidelines that promote use of urine drug testing, state prescription drug monitoring programs, and patient provider agreements (PPAs). ${ }^{2,3}$

Since the 1970s, PPAs have been recommended as one opioid risk mitigation tool. ${ }^{4}$ The Food and Drug Administration recommends PPAs to "ensure the appropriate and safe use of opioid medicines." In their review, the CDC found no studies on PPA effectiveness but suggested they could improve safety by clarifying treatment expectations. ${ }^{2}$ Two systematic reviews ${ }^{6,7}$ of low- to moderate-quality studies did not find an association between use of PPAs and rates of opioid misuse. PPA content and processes vary widely with no consensus on the appropriate content, length, or manner of administration, making it difficult to measure effectiveness.

There are many provider-related barriers to administering PPAs, including time pressure and competing priorities, which can make implementing PPAs more of a pro forma task than a meaningful patient education process. Patients may also find it difficult to comprehend PPAs and may view it simply as another form to be signed. A study ${ }^{8}$ examining PPA readability from pain specialists found them written at a mean 14th grade reading level, far above the recommended fifth-grade reading level. ${ }^{9}$ Reports on the thematic content of PPAs are nearly two decades old. ${ }^{10}$ It is important to understand what PPAs communicate to patients since they can impact the patient-provider relationship. ${ }^{11}$ They can be viewed as coercive by patients who are faced with the choice of signing the PPA or being denied opioid analgesics. ${ }^{12}$ These patients may feel stigmatized since PPAs are not required for other 
treatments that can also cause morbidity and mortality (e.g., warfarin, insulin). ${ }^{13-15}$

To address these gaps in the literature, we conducted a national survey and collected PPAs to achieve the following aims: (1) describe processes of administering PPAs, (2) assess provider views on the utility of PPAs, and (3) assess the readability and thematic content of a national sample of PPAs.

\section{METHODS}

\section{Population}

Electronic surveys with requests for copies of PPAs were sent to a convenience sample of a national registry of 62,530 individuals who had registered for Boston University's Safer/Competent Opioid Prescribing Education (SCOPE of Pain) program between March 2013 and June 2017 and did not opt out of the SCOPE of Pain mailing list. SCOPE of Pain is a series of continuing education activities on safer opioid prescribing for pain. ${ }^{16}$ Registrants comprise a diverse population including physicians, advanced practice nurses, registered nurses, and dentists who may or may not prescribe opioids for chronic pain. Our goal was to receive responses from a geographically diverse cohort of individuals who use PPAs when prescribing opioids for chronic pain. Three email rounds were sent over the course of 1 month (July 2017) requesting participation in the electronic survey plus an option to upload their PPA.

\section{Electronic Survey}

Only respondents who use PPAs when prescribing opioids for chronic pain were invited to complete the survey. The 15question survey asked about three practice characteristics: state in which the practice is located, primary specialty (e.g., primary care, pain care), and practice type (solo, group, academic, hospital-based, VA, or other). Participants were asked the origin of their PPA; person(s) who typically administers the PPA with patients; frequency with which patients sign the PPA; average perceived time spent administering a PPA; and whether their clinic and/or state requires PPAs for patients prescribed opioids for chronic pain. Participants were asked to rate (often, always, sometimes, never, or rarely) whether PPAs are effective in reducing opioid misuse and whether PPAs are "worth the effort."

Participants from addiction specialty settings were excluded because their PPAs were used for addiction treatment rather than pain treatment. Statistical analysis consisted of frequency distributions for categorical variables and measures of central tendency for numerical variables. The Boston University Medical Campus Institutional Review Board (IRB) determined this evaluation to be exempt from further IRB review.

\section{Patient Provider Agreements}

Readability Analysis. PPAs used for prescribing opioids for chronic pain were included while those used by addiction specialists were excluded. PPAs were assessed for readability using two validated readability measures, the Flesch-Kincaid Grade Level and the New Dale-Chall Readability Formula. The Flesch-Kincaid Grade Level is based on the average sentence length in words and average number of syllables per word. ${ }^{17}$ The New Dale-Chall Formula is based on the average sentence length in words and the percentage of "difficult words" compared to a list of familiar words. ${ }^{18}$

To make the PPAs suitable for the readability formulas, they were converted into Microsoft ${ }^{\circledR}$ Word Documents; end punctuation was added to section headings and bulleted lists; institution names and proper nouns were standardized; and medication brands were changed to generic drug names. Only PPAs with text organized in sentences were included in readability analyses. Frequency distributions were calculated for each readability measure, as well as the mean and range word count for submitted PPAs.

Thematic Analysis. Two researchers (JL and KP) systematically reviewed 10 submitted PPAs, documented concepts present, and then met with two additional team members (DA and MPO) who have extensive clinical and research experience in safer opioid prescribing. Together, the team reviewed the initial notes and drafted a preliminary codebook. Ten randomly selected PPAs were double-coded, followed by a meeting to resolve coding discrepancies and clarify code meanings. The two researchers then each coded half of the PPAs. Midway through, the research team met to revise the codebook and delete, add, merge, and refine codes; the final codebook was then used to code all PPAs. The research team categorized the codes into major themes based on their clinical experience using PPAs and selected the most clinically informative codes within major themes for data display, excluding those that addressed clinic-specific logistics (e.g., who to call for refills). For analysis, we calculated the frequency of each code in the PPA sample.

\section{RESULTS}

\section{Electronic Survey Results (Table 1)}

Among 62,530 individuals in the SCOPE of Pain registry contacted for the study, 926 responded with 443 reporting PPA use. Thirteen respondents were excluded because they worked in addiction treatment settings. The final analytical sample of 430 respondents represented 43 states with $50 \%$ coming from New York, Massachusetts, California, Michigan, and North Carolina. Most reported their specialty as primary care (e.g., internal medicine, family medicine) $(64 \%)$ or pain care $(18 \%)$ and represented a range of practice types including group (34\%), solo (20\%), academic (17\%), and hospital-based (16\%) practices.

Among PPA users, $41 \%$ created an original PPA while 30\% modified a pre-existing one. Most respondents reported that 
Table 1 Survey on Patient Provider Agreement (PPA) Use $(n=430)$

\begin{tabular}{ll}
\hline \hline Survey question & $\boldsymbol{n}(\boldsymbol{\%})$ \\
\hline Origin of PPA & \\
Original & $174(41)$ \\
Pre-existing PPA with modifications & $130(30)$ \\
Pre-existing PPA, no modifications & $27(6)$ \\
Other & $28(6)$ \\
Unknown & $71(17)$ \\
Frequency of patient signing PPA & $178(41)$ \\
Once & $171(40)$ \\
Annually & $19(4)$ \\
More than annually & $62(14)$ \\
Other & $343(80)$ \\
Person(s) who administers PPA* & $130(30)$ \\
Prescriber & $18(4)$ \\
Nurse & $63(15)$ \\
Pharmacist & \\
Other & $314(73)$ \\
PPA required by clinic? & $87(20)$ \\
Required & $29(7)$ \\
Recommended & $113(26)$ \\
Other & $155(36)$ \\
PPA required by state law? & $162(38)$ \\
Yes & \\
No & $14(28)$ \\
Unsure & $214(50)$ \\
How often do PPAs reduce opioid misuse? & $96(22)$ \\
Often or always & $285(66)$ \\
Nometimes & $114(27)$ \\
How often are these agreements worth the effort? & $31(7)$ \\
Often or always & \\
Sometimes & \\
Never or rarely & \\
\hline &
\end{tabular}

*Could select more than one option

patients signed their PPA either once (41\%) or annually (40\%). Prescribers were most commonly responsible for administering PPAs $(80 \%)$ with patients, followed by nurses (30\%). Overall, respondents perceived administering PPAs as time-intensive with an average perceived time of $13 \mathrm{~min}$ (range, 10 to $70 \mathrm{~min}$ ). PPAs were more commonly required by respondents' institution (73\%) than by state law (26\%). However, $38 \%$ were uncertain whether their state mandated PPAs. While $66 \%$ of respondents reported that PPAs are "often" or "always" worth the effort, only $28 \%$ indicated they are "often" or "always" effective in reducing opioid misuse.

\section{Patient Provider Agreement Results}

Readability (Fig. 1). Among the 128 PPAs submitted, six from addiction specialists were excluded. One was excluded from only the readability analysis due to insufficient prose. The remaining 121 PPAs represented 34 states with similar specialty and practice type distributions as the survey sample.

The word count of submitted PPAs ranged from 125 to 4350 (mean 881). The Flesch-Kincaid reading level ranged from fourth grade to college and above, with most PPAs written at high school level or beyond (Fig. 1). Only 2.5\% (3/121) were at or below a fifth-grade reading level. The New Dale-Chall readability results were consistent with the FleschKincaid findings with most PPAs written at high school level and only $1.7 \%(2 / 121)$ written at or below the fifth to sixth grade range.
Thematic Analysis (Table 2). Codes from the 122 analyzed PPAs fell under seven major themes: rules to reduce misuse; monitoring for misuse; consequences of non-adherence or misuse; prescription policies; education on opioid risks; treatment plan and goals; and patient rights (Table 2). Within the rules to reduce misuse theme, $81 \%$ of PPAs mandated abstinence from illegal drugs, and $62 \%$ recommended avoiding alcohol use. Cannabis was explicitly banned in 19 PPAs $(16 \%)$, and one PPA recommended discussing legal cannabis use with their provider. Monitoring for misuse included drug tests (92\%), pill counts (48\%), and prescription drug monitoring programs $(27 \%)$. PPAs commonly focused on punitive consequences of non-adherence; for example, $85 \%$ discussed grounds for discontinuing opioid prescriptions and 51\% warned patients could be discharged from care for non-adherence. PPAs commonly (47\%) authorized providers to cooperate with law enforcement if the patient engaged in illegal activity.

Nearly all PPAs discussed prescription policies, including mandating a single prescriber $(87 \%)$ or single pharmacy (75\%), banning early refills (69\%), and/or consequences of lost or stolen medications (85\%). Sections devoted to these policies were typically framed as warnings that providers would not prescribe additional opioids in these circumstances.

Within the education on opioid risks theme, $79 \%$ mentioned physical dependence and/or withdrawal, $72 \%$ risk of addiction, and 57\% risk of overdose. Over half of PPAs discussed some aspect of the patient's treatment plan and goals of opioid therapy, including $62 \%$ that encouraged or required nonopioid treatment modalities for pain. The theme of patient rights was mentioned in only $7 \%$ of PPAs, including the patient's right to terminate the agreement. Three PPAs mentioned a pain care "bill of rights" that included rights to shared decision-making and respectful and non-discriminatory care. $^{19}$

\section{DISCUSSION}

In this national survey of providers who use patient provider agreements (PPAs) when prescribing opioids for chronic pain, PPAs are primarily prescriber-implemented and a timeintensive process. While $66 \%$ of respondents viewed PPAs as "often" or "always" worth the effort, only $28 \%$ considered them "often" or "always" effective in reducing opioid misuse. The reading burden of submitted PPAs far surpassed recommended standards for patient education materials with $93 \%$ of PPAs written at high school level or higher. Finally, PPAs often had a punitive tone, focusing on consequences of nonadherence, including prescription discontinuation $(85 \%)$ and discharge from care (51\%). Less attention was paid to provider responsibilities (e.g., keeping the patient safe, ensuring timely refills), treatment goals, and patient rights. While $72 \%$ of PPAs mentioned addiction as a risk of opioids, only $39 \%$ 


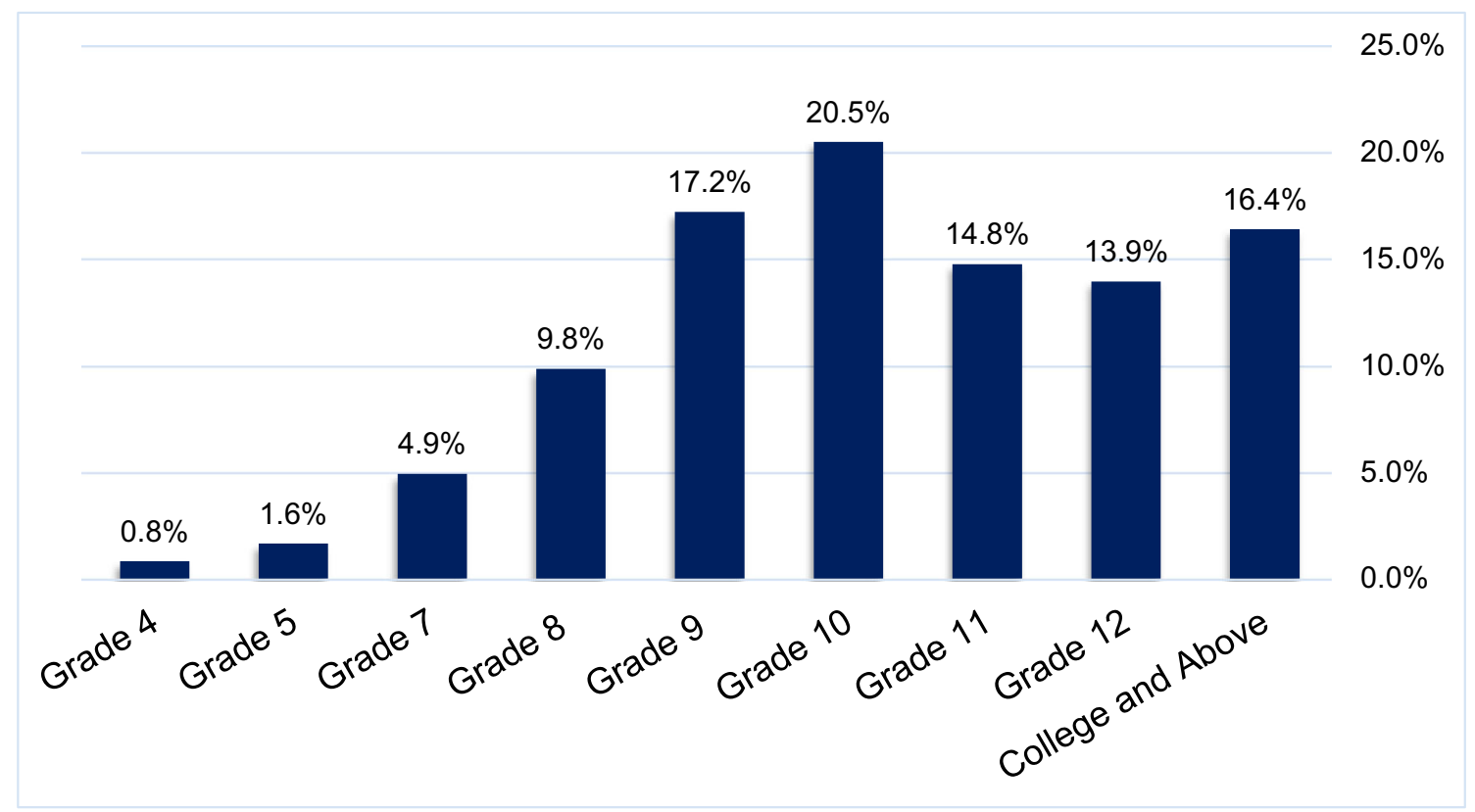

Figure 1 Flesch-Kincaid grade level of patient provider agreement (PPA) samples $(n=121)$.

discussed the provider's role in connecting the patient to addiction treatment.

Survey respondents expressed greater confidence in PPAs' utility than in their efficacy in reducing opioid misuse, consistent with a 2015 provider survey. ${ }^{20} \mathrm{~A}$ 2010 systematic review evaluating the association of PPAs with opioid misuse found poor- to fair-quality observational studies with wide variation in PPAs and opioid misuse measures. ${ }^{6}$ Among the four studies with comparison groups, multi-component interventions that included a PPA were associated with a $7 \%$ to $23 \%$ reduction in misuse outcomes. ${ }^{6}$ A systematic review 10 years later found that low- to moderate-quality studies did not show a consistent association between PPAs and opioid misuse outcomes. ${ }^{7}$ The one randomized clinical trial evaluating the effects of a multi-component management strategy that included a PPA found an association with improved guideline adherence but not with decreased early refills, a commonly used indicator of opioid misuse. ${ }^{21}$

Despite the limited evidence base, healthcare providers may view PPAs as valuable for purposes other than reducing opioid misuse, including obtaining informed consent, communicating clinic policies, and mitigating legal risk. In a 2014 survey of primary care physicians, most believed PPAs were useful to protect physicians but few believed they prevented opioid misuse. $^{22}$ This supports the idea that current PPAs may primarily serve providers, not patients.

Our findings indicate modestly improved PPA readability compared to that reported in 2007 by Roskos et al., who found PPAs from American Pain Society members to be written at a mean 14th grade reading level. ${ }^{8}$ Regardless of this progress, current PPAs remain inaccessible for most of the population as half of US adults have limited or low health literacy levels, and the average adult English reading level is eighth to ninth grade. ${ }^{23}$ In 2010, the Joint Commission recommended that patient education materials be written at or below a fifth-grade reading level. ${ }^{9}$ Thus, most patients who sign PPAs likely do not understand them, raising concern about whether this process is meaningful or fair. A qualitative study ${ }^{24}$ of patient and provider perceptions of PPAs supports this concern. Patients exhibited low awareness of signing a PPA and insufficient understanding of its purpose and content, confusing PPAs with other forms they were asked to sign during the visit (e.g., HIPAA consent).

The length of submitted PPAs varied substantially. While we are unaware of a standard recommendation for length of patient education materials, we know that excessively long documents can be daunting. New evidence suggests that word count and passage length increase reading difficulty independent of task complexity because long text sections appear more demanding and result in more mind-wandering. ${ }^{25}$ Longer documents with text uninterrupted by bullets or numbering may be less effective educational tools. Based on the estimated reading speed of 200 words per minute for individuals who read at a ninth grade level, ${ }^{26}$ a PPA with the mean length of 881 words would require about 4 min of sustained silent reading, in addition to time needed to answer questions. Notably, we did not determine how the submitted PPAs were administered (e.g., read by patients, read aloud by clinicians, or neither), which would influence duration and could improve comprehension and adherence.

Our thematic analysis builds upon a 1999 qualitative study of PPAs from 39 pain management programs. ${ }^{10}$ In that study, two statement categories were mentioned in over $90 \%$ of 
Table 2 Thematic Analysis of Patient Provider Agreement (PPA) Samples $(n=122)$

\begin{tabular}{|c|c|c|}
\hline Major themes & Clinically important codes & $\begin{array}{l}n \\
(\%)\end{array}$ \\
\hline \multirow[t]{4}{*}{ Rules to reduce misuse } & $\begin{array}{l}\text { No medication selling, trading, or } \\
\text { sharing }\end{array}$ & $\begin{array}{l}104 \\
(84)\end{array}$ \\
\hline & No illegal drugs & $\begin{array}{l}99 \\
(81)\end{array}$ \\
\hline & Avoid alcohol & 76 \\
\hline & $\begin{array}{l}\text { No consumption of others' } \\
\text { prescriptions }\end{array}$ & $\begin{array}{l}39 \\
(32)\end{array}$ \\
\hline \multirow[t]{3}{*}{ Monitoring for misuse } & Drug tests & $\begin{array}{l}112 \\
(92)\end{array}$ \\
\hline & Pill counts & $\begin{array}{l}59 \\
(48)\end{array}$ \\
\hline & $\begin{array}{l}\text { Prescription drug monitoring } \\
\text { program }\end{array}$ & $\begin{array}{l}33 \\
(27)\end{array}$ \\
\hline \multirow[t]{4}{*}{$\begin{array}{l}\text { Consequences of non- } \\
\text { adherence or misuse }\end{array}$} & $\begin{array}{l}\text { Grounds for prescription } \\
\text { discontinuation }\end{array}$ & $\begin{array}{l}104 \\
(85)\end{array}$ \\
\hline & $\begin{array}{l}\text { Discharge from care due to non- } \\
\text { adherence }\end{array}$ & $\begin{array}{l}62 \\
(51)\end{array}$ \\
\hline & $\begin{array}{l}\text { Cooperation with law } \\
\text { enforcement permissible if } \\
\text { evidence of illegal activity }\end{array}$ & $\begin{array}{l}57 \\
(47)\end{array}$ \\
\hline & $\begin{array}{l}\text { Referral to addiction treatment if } \\
\text { appropriate }\end{array}$ & $\begin{array}{l}47 \\
(39)\end{array}$ \\
\hline \multirow[t]{4}{*}{ Prescription policies } & Single prescriber only & $\begin{array}{l}106 \\
(87)\end{array}$ \\
\hline & Single pharmacy only & $\begin{array}{l}92 \\
(75)\end{array}$ \\
\hline & No early refills & $\begin{array}{l}84 \\
(69)\end{array}$ \\
\hline & $\begin{array}{l}\text { Management of lost or stolen } \\
\text { medications }\end{array}$ & $\begin{array}{l}104 \\
(85)\end{array}$ \\
\hline \multirow{6}{*}{$\begin{array}{l}\text { Education on opioid } \\
\text { risks }\end{array}$} & Physical dependence or & 96 \\
\hline & withdrawal & (79) \\
\hline & Addiction & $\begin{array}{l}88 \\
(72)\end{array}$ \\
\hline & Side effects & $\begin{array}{l}73 \\
(60)\end{array}$ \\
\hline & Overdose & $\begin{array}{l}70 \\
(57)\end{array}$ \\
\hline & Death & $\begin{array}{l}62 \\
(51)\end{array}$ \\
\hline \multirow[t]{5}{*}{$\begin{array}{l}\text { Treatment plan and } \\
\text { goals }\end{array}$} & $\begin{array}{l}\text { Inclusion of non-opioid treatment } \\
\text { modalities }\end{array}$ & $\begin{array}{l}76 \\
(62)\end{array}$ \\
\hline & $\begin{array}{l}\text { Goal to improve function or } \\
\text { quality of life }\end{array}$ & $\begin{array}{l}70 \\
(57)\end{array}$ \\
\hline & Collaboration with other & 59 \\
\hline & providers & $(48)$ \\
\hline & $\begin{array}{l}\text { Re-evaluation of opioid therapy } \\
\text { effectiveness }\end{array}$ & $\begin{array}{l}51 \\
(42)\end{array}$ \\
\hline \multirow[t]{2}{*}{ Patient rights } & Right to terminate agreement & $9(7)$ \\
\hline & Pain care bill of rights & $4(3)$ \\
\hline
\end{tabular}

PPAs: requirements to avoid misuse of controlled substances and terms of prescription termination. ${ }^{10}$ Other common themes similar to our findings included mandatory drug screens and restriction to a single prescriber. Similarities in our analyses suggest that after two decades, PPAs are still provider-centric and focused on establishing and enforcing rules. Threats of prescription termination or discharge from care communicate to patients that if they exhibit behaviors suggestive of medication misuse, their provider may end the care relationship. Additionally, most PPAs do not address the continuation of opioid analgesics for patients who use cannabis, an important unaddressed concern in the context of increasing legal availability of medical and recreational cannabis.
Strengths of our study include the geographic and practice type diversity of our sample and the use of common validated readability assessments. Nonetheless, there are several limitations. First, our 1.5\% $(926 / 62,530)$ response rate was low. This was likely due to the use of a convenience sample of individuals who registered for a safer opioid prescribing education program, many of whom do not prescribe opioids for chronic pain and/or do not practice in settings where PPA use is indicated (e.g., emergency and inpatient settings). We do not know if our pool of respondents reflects a representative sample of clinicians who use PPAs. Selection bias may have played a role, as survey respondents may have had greater interest in chronic pain management than the national population of PPA users. Additionally, providers with salient positive or negative experiences with PPAs may have been more inclined to participate. Social desirability bias may have influenced which respondents submitted PPAs. We presume that respondents would be more likely to submit a PPA if they were proud, or at least not ashamed, of their practice's PPA. This would bias results toward higher quality PPAs, suggesting that the true national population of PPAs may be worse than those analyzed.

Finally, there are limitations inherent to computerized readability assessment formulas. The Flesch-Kincaid Scale is wellvalidated and widely available since it is embedded in Microsoft@ Word. The New Dale-Chall Formula correlates well with the Flesch-Kincaid Scale but also incorporates a measure of word familiarity. ${ }^{17,18}$ However, readability tests do not identify what people understand, and they typically underestimate the reading level required to understand medical texts that involve complex concepts. Additionally, although we measured document length using word count, we did not evaluate other formatting characteristics that affect readability ${ }^{27}$ such as font size and type, headings, and layout, and we did not directly assess the amount of time that would reasonably be needed to review PPAs.

There remain many unanswered questions about PPA use. We only surveyed providers who used PPAs and did not inquire why some individuals choose not to use PPAs. Additionally, our study did not collect data on participant demographics (e.g., age, gender, race/ethnicity, years in practice) or compare PPAs from different geographic regions and practice types. Future studies should address these important questions.

National organizations recommend PPAs for patients prescribed opioids for chronic pain and increasing numbers of state licensing boards and payers now require them. As a result, we are investing substantial resources into PPAs without knowing if they are effective. We need to determine which components of PPAs are most effective and, from there, develop a high-quality standardized PPA with guidelines for administering it with patients. Widespread adoption of PPAs based on a national standard would permit rigorous analysis of their effects on patient outcomes, including prescription opioid misuse and achievement of treatment goals, and provider outcomes, such as satisfaction and mastery. 
If we continue to use PPAs in the absence of strong evidence, we should use meaningful, bidirectional, and readable documents, rather than provider-centered "contracts". ${ }^{11}$ PPAs should encourage shared decision-making and avoid coercive or pejorative language. Additional PPA design strategies have been proposed for patients with low health literacy such as incorporating more visual or interactive content. ${ }^{8}$

We have an opportunity to transform PPAs from a regulatory step that risks eroding patient-provider trust into a meaningful tool that promotes a therapeutic alliance in chronic pain management. We should move forward in developing an understandable, non-stigmatizing, reciprocal gold standard PPA that can be widely disseminated and studied.

Corresponding Author: Jordana Laks, MD, MPH; Section of General Internal Medicine, Department of Medicine, Boston University School of Medicine and Boston Medical Center, Boston, MA, USA (e-mail: jordana.laks@bmc.org).

\section{Compliance with Ethical Standards:}

Conflict of Interest: The authors declare that they do not have a conflict of interest.

\section{REFERENCES}

1. Dart RC, Surratt HL, Cicero TJ, et al. Trends in opioid analgesic abuse and mortality in the United States. N Engl J Med. 2015;372(3):241-8.

2. Dowell D, Haegerich TM, Chou R. CDC Guideline for prescribing opioids for chronic pain-United States, 2016. MMWR Recomm Rep. 2016;65(1): 1-49.

3. US Department of Veterans Affairs. VA/DoD clinical practice guidelines: management of opioid therapy for chronic pain. Available at: https:// www.healthquality.va.gov/guidelines/Pain/cot/VADoDOTCPG022717. pdf Accessed February 8, 2020.

4. Fishman SM, Kreis PG. The opioid contract. Clin J Pain. 2002;18(4):S70-S75.

5. Food and Drug Administration (FDA). Opioid Patient Prescriber Agreement (PPA). https://www.fda.gov/media/114694/download. Accessed June 27, 2020.

6. Starrels JL, Becker WC, Alford DP, Kapoor A, Williams AR, Turner BJ. Systematic review: treatment agreements and urine drug testing to reduce opioid misuse in patients with chronic pain. Ann Intern Med. 2010;152(11):712-20.

7. McAuliffe Staehler TM, Palombi LC. Beneficial opioid management strategies: A review of the evidence for the use of opioid treatment agreements. Subst Abus. 2020;3:1-8.

8. Roskos SE, Keenum AJ, Newman LM, Wallace LS. Literacy demands and formatting characteristics of opioid contracts in chronic nonmalignant pain management. J Pain. 2007;8(10):753-8.
9. Advancing Effective Communication, Cultural Competence, and Patient and Family-Centered Care: A Roadmap for Hospitals. The Joint Commission. Oakbrook Terrace, IL; 2010.

10. Fishman SM, Bandman TB, Edwards A, Borsook D. The opioid contract in the management of chronic pain. J Pain Symptom Manag. 1999; 18(1):27-37.

11. Tobin DG, Forte KK, McGee SJ. Breaking the pain contract: a better controlled substance agreement for patients on chronic opioid therapy. Cleve Clin J Med. 2016;83(11):827-35.

12. Albrecht JS, Khokhar B, Pradel F, et al. Perceptions of patient provider agreements. J Pharm Health Serv Res. 2015;6(3):139-44.

13. De Ruddere L, Craig KD. Understanding stigma and chronic pain: astate-of-the-art review. Pain. 2016;157(8):1607-10.

14. Upshur CC, Bacigalupe G, Luckmann R. "They don't want anything to do with you": patient views of primary care management of chronic pain. Pain Med. 2010;11(12):1791-8.

15. Frantsve LM, Kerns RD. Patient-provider interactions in the management of chronic pain: current findings within the context of shared medical decision making. Pain Med. 2007;8(1):25-35.

16. Alford DP, Zisblatt L, Ng P, Hayes SM, Peloquin S, Hardesty I, White JL. SCOPE of Pain: an evaluation of an opioid risk evaluation and mitigation strategy continuing education program. Pain Med 2016;17(1):52-63.

17. The Flesch Grade Level readability formula. Available at: www. readabilityformulas.com/flesch-grade-level-readability-formula.php. Accessed February 8, 2020.

18. New Dale-Chall readability formula. Available at: www. readabilityformulas.com/new-dale-chall-readability-formula.php. Accessed February 8, 2020.

19. US Pain Foundation. Patient Bill of Rights. Available at: https:// uspainfoundation.org/wp-content/uploads/2017/11/patient-bill-ofrights-combined.pdf. Accessed February 8, 2020.

20. N Kraus C, T Baldwin A, A Curro F, McAllister RG. Clinical implications of patient-provider agreements in opioid prescribing. Curr Drug Saf. 2015; 10(2): 159-64.

21. Liebschutz JM, Xuan Z, Shanahan CW, et al. Improving adherence to long-term opioid therapy guidelines to reduce opioid misuse in primary care: a cluster-randomized clinical trial. JAMA Intern Med. 2017;177(9): 1265-1272.

22. Starrels JL, Wu B, Peyser D, et al. It made my life a little easier: primary care providers' beliefs and attitudes about using opioid treatment agreements. J Opioid Manag. 2014;10(2):95-102.

23. Davis TC, Wolf MS. Health literacy: implications for family medicine. Fam Med. 2004;36(8):595-8.

24. Penko J, Mattson J, Miaskowski C, Kushel M. Do patients know they are on pain medication agreements? Results from a sample of high-risk patients on chronic opioid therapy. Pain Med. 2012;13(9):1174-80.

25. Forrin ND, Risko EF, Smilek D. On the relation between reading difficulty and mind-wandering: a section-length account. Psychol Res. 2019;83:485-97.

26. Carver RP. Silent reading rates in grade equivalents. J Reading Behavior. 1989;21(2):155-66.

27. Peterson BT, Clancy SJ, Champion K, McLarty JW. Improving readability of consent forms: what the computers may not tell you. IRB. 1992;14:6-8.

Publisher's Note: Springer Nature remains neutral with regard to jurisdictional claims in published maps and institutional affiliations. 